\title{
Inhaltsverzeichnis des ersten Bandes
}

\author{
(\$S 1-104)
}

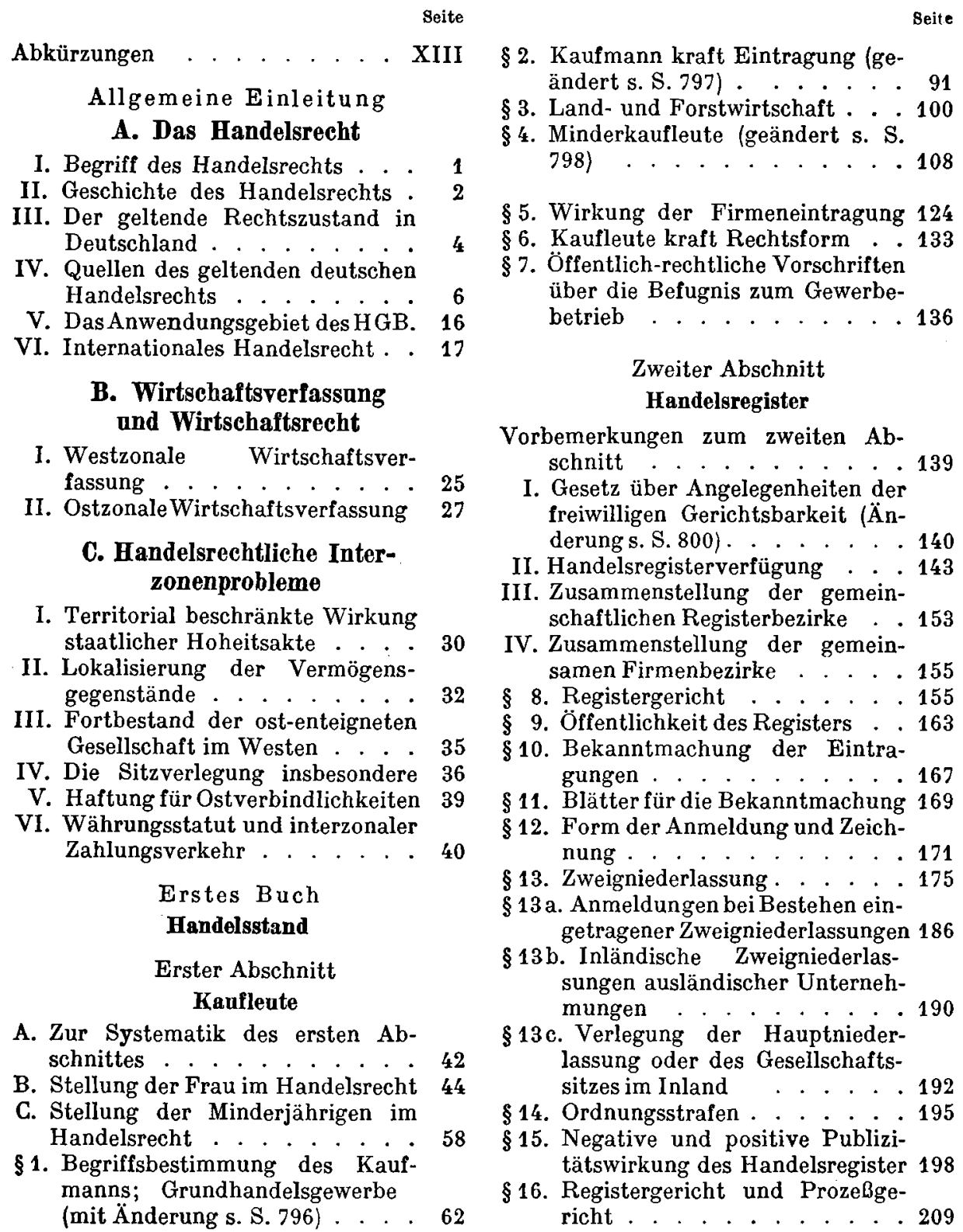


Seito

\section{Dritter Abschnitt}

\section{Handelsfirma}

Vorbemerkungen . . . . . . . . . 214

§ 17. Begriff und Bedeutung d. Firma 215

\$18. Firma eines Einzelkaufmannes . 233

§ 19. Firma einer OGH. und KoGes. 246

$\S 20$. Firma einer AG. und KoAG. . . 250

$\S 21$. Fortführung der Firma bei Namensänderung . . . . . . . 250

§22. Fortführung der Firma bei Geschäftsübernahme . . . . . . 252

$\S 23$. Keine Veräußerung der Firma ohne Übertragung des Handelsgeschäfts . . . . . . . . 289

§24. Übergang des Firmenrechts bei teilweisem Wechsel d. Geschäftsinhaber . . . . . . . . 290

$\S 25$. Verhältnis des Geschäftserwerbers zu Geschäftsgläubigern und -schuldnern . . . . . . . . . 293

$\S 26$. Verjährung zugunsten des $\mathrm{Ge}-$ schäftsveräußerers . . . . . . 307

$\S 27$. Haftung der Erben für die Geschäftsverbindlichkeiten . . . 311

§28. Eintritt in das Geschäft eines Einzelkaufmannes . . . . . 322

$\S 2$. Pflicht zur Anmeldung u. Zeichnung der Firma . . . . . . . . 327

§30. Firmenverschiedenheit . . . . 329

§31. Änderung und Erlöschen der Firma; Verlegung der Niederlassung . . . . . . . . . . . 337

§ 32. Eintragungen im Konkursfalle . 343

$\S 33$. Anmeldung gewisser juristischer Personen . . . . . . . . . . 344

§34. Änderung in den Rechtsverhältnissen der juristischen Personen 348

§35. Zeichnung der Unterschrift bei einer juristischen Person. . . . 351

§36. Gewerbliche Unternehmungen öffentlicher Körperschaften . . 351

§ 37. Unbefugter Firmengebrauch . . 355

A. Die öffentlich-rechtl. Folge . 355

B. Die privat-rechtl. Folgen . . 361

C. Verhältnis des Firmenrechts zum Warenzeichenrecht . . 370

D. Verhältnis des Firmenrechts zum Wettbewerbsrecht. . . 371

E. Ergänzung des Firmenrechts durch die Gewerbeordnung . 373

Vierter Abschnitt

Handelsbïcher

Vorbemerkungen: Richtlinien zur Organisation der Buchführung . . 376
§38. Handelsrechtliche Buchführungspflicht . . . . . . 387

$\S 39$. Pflicht zur Aufstellung von Inventar und Bilanz . . . . . . 393

$\S 40$. Wertansatz in der Bilanz . . 401

\$41. Unterzeichnung von Inventar und Bilanz . . . . . . . . 406

$\S 42$. Rechnungsabschlüsse öffentlicher Körperschaften ․ . . . 408

§43. Form der Handelsbücher . . . 411

$\S 44$. Aufbewahrungspflicht . . . . 416

\$45. Vorlegung im Rechtsstreit . . . 418

$\$ 46$. Umfang der Offenlegung . . . 421

$\S 47$. Offenlegung bei Vermögensauseinandersetzungen . . . . . . 422

Fünfter Abschnitt

Prokura und Handlungsvollmacht

Vorbemerkungen über Vollmacht im Allgemeinen . . . . . . . 424

$\S 48$. Erteilung der Prokura . . . 460

\$ 49. Umfang der Prokura . . . . . 469

$\S 50$. Unbeschränkbarkeit d.Umfangs 473

$\S 51$. Zeichnung durch d. Prokuristen 476

§52. Widerruf, Unübertragbarkeit, Erlöschen der Prokura . . . . 477

§53. Eintragung der Prokura u. ihres Erlöschens . . . . . . . . . 479

$\S 54$. Begriff und Umfang der Handlungsvollmacht . . . . . . . 482

$\S 55$. Handlungsreisende (außer Kraft s. S. 669). . . . . . 492

$\S 56$. Angestellte im Laden od. offenen Warenlager . . . . . 495

$\S 57$. Zeichnung durch den Handlungsbevollmächtigten . . . . 497

$\S 58$. Übertragung der Vollmacht . 498

$$
\text { Sechster Abschnitt }
$$

Handlungsgehilfen und Handlungslehrlinge

Vorbemerkungen . . . . . . . . 499

$\S 59$. Begriff. Leistungen u. Ansprüche 501

$\S 60$. Gesetzliches Wettbewerbsverbot 545

§61. Folgen der Verletzung. . . . . 549

$\S 62$. Fürsorgevorschriften . . . . . 552

$\S 63$. Dienstbehinderung durch unverschuldetes Unglück . . . 560

§ 64. Gehaltszahlung . . . . . . . 567

$\S 65$. Provision (geändert; s. S.673). . 568

$\S 66$. Gesetzliche Kündigungsfrist . . 568

$\S 67$. Vereinbarte Kündigungsfrist . . 580

$\S 68$. Gehilfen mit höherem Gehalt oder in außereuropäischer Niederlassung . . . . . . . 584 
§ 69. Vorübergehende Aushilfe . . . 586

$\S 70$. Fristlose Kündigung . . . . . 588

$\S 71$. Wichtige Kündigungsgründe des 598 Gehilfen . . . . . . . 598

$\S 72$. Wichtige Kündigungsgründe des Prinzipals . . . . . . . . . 600

§73. Zeugnis . . . . . . . . . . 611

$\$ 74$. Vertragliches Wettbewerbsverbot . . . . . . . . . 614

\$74 a Unverbindlichkeit und Nichtigkeit . . . . . . . . . 621

$\S 74$ b. Karenzentschädigung . . . . 626

$\$ 74 \mathrm{c}$. Anrechnung anderweitigen Erwerbs. 629

$\S 75$. Wegfall des Wettbewerbsverbotes . . . . . . . . . 631

$\S 75$ a. Verzicht auf das Verbot . . 635

$\S 75$ b. Ausnahmen . . . . . . . . 637

$\S 75$ c. Fall der Vertragsstrafe . . . . 639

$\S 75 \mathrm{~d}$. Umgehungen . . . . . . . . 643

\$75e. Konkursvoranrecht der Karenzentschädigung . . . . . . . 644

$\S 75 \mathrm{f}$. Geheime Konkurrenzabrede . 645

$\S 75 \mathrm{~g}$ u. $\S 75 \mathrm{~h}$ s. Siebenter Abschnitt 674

$\S 76$. Pflichten des Lehrherrn und des Lehrlings . . . . . . . . 646

§ 77. Dauer des Lehrvertrags . . . . 654

$\S 78$. Übergang zu einem anderen $\mathrm{Ge}$ werbe oder Beruf 657

$\S 79$. Unbefugter Austritt . . . . . .660

$\S 80$. Zeugnis . . . . . . . . . . . 662

§ 81. Bürgerliche Ehrenrechte des Lehrherrn . . . . . . 663

\$ 82. Strafvorschriften

$\$ 82 \mathrm{a}$. Wettbewerbsabrede von Volontären . . . . . . 665

§ 83. Andere Angestellte . . . . . . 666

\section{Siebenter Abschnitt}

\section{Handelsvertreter}

I. Einleitung: Das Handelsvertre-

II. Die Vorschriften des Handelsvertretergesetzes . . . . . . 668

§ 55. Umfang der Vollmacht . . . . . 669

$\S 65$. Provision angestellter Geschäftsmittler . . . . . . . . 674

$\$ 75 \mathrm{~g}$. Ermächtigung des reisenden Angestellten

$\S 75 \mathrm{~h}$. Abschluß durch nichtbevollmächtigten angestellten Geschäftsmittler . . . . . . . . 675

III. Vorbemerkungen vor $\$ 84$. . 676

§ 84. Begriff Handelsvertreter . . 680
§ 85. Beurkundung des Vertragsin-

halts . . . . . . . . 700

$\S 86$. Pflichten des Handelsvertreters 700

$\$ 86$ a. Pflichten des Unternehmers . . 704

$\$ 86$ b. Delcredere . . . . . . . 706

$\S 87$. Kreis der provisionspflichtigen Geschäfte, Bezirksvertreter, Inkassoprovision . . . . . 709

$\$ 87 \mathrm{a}$. Voraussetzungen der Entstehung d. Provisionsanspruches 720

$\S 87 \mathrm{~b}$. Höhe und Berechnung der Provision . . . . . . . . 225

$\S 87$ c. Abrechnung über die Provision

Buchauszug . . . . . . . 727

$\S 87$ d. Spesenersatz . . . . . . . 730

\$ 88. Verjährung der Ansprüche . . . 731

$\$ 88$ a. Kein Verzicht in voraus auf Zurückbehaltungsrechte. . . . 731

$\S 89$. Ordentliche Kündigung . . . . 732

$\$ 89$ a. Fristlose Kündigung aus wichtigem Grunde . . . . . . . . 738

$\S 89 \mathrm{~b}$. Ausgleichsanspruch des Handelsvertreters . . . . . . . 742

$\S 90$. Verpflichtung zur Geheimniswahrung . . . . . . . 752

$\S 90$ a. Wettbewerbsabrede . . . . 753

$\S 91$. Erstreckung des $\S 55 ;$ Ermächtigung des Vermittlungsvertreters........ . 756

$\S 91$ a. Geschäftsabschluß durch Vermittlungsvertreter . . . . 757

\$ 92. Versicherungsvertreter . . . 762

\$ 92 a. Abhängiger Einfirmenvertreter 767

$\S 92$ b. Handelsvertreter im Nebenberuf . . . . . . . . . . 769

§92c. Auslandsvertreter, Transportund Schiffahrtsvertreter. . . . 772

\section{Achter Abschnitt}

\section{Handelsmäkler}

Vorbemerkungen: der Zivilmäklervertrag . . . . . . 774

§ 93. Begriff. . . . . . . . . 782

$\$$ 94. Schlußnote . . . . . . . 785

§ 95. „Aufgabevorbehalte“ . . . . 787

$\S$ 96. Aufbewahrung der Proben . . 791

$\$$ 97. Umfang der Vollmacht . . . 791

$\S$ 98. Schadenshaftung . . . . . . 792

§ 99. Mäklerlohn. . . . . . . . . 792

$\$$ 100. Tagebuch . . . . . . . . 793

$\S 101$. Auszüge aus dem Tagebuch . . 794

$\S 102$. Vorlegung des Tagebuches im Rechtsstreit . . . . . 795

§103. Strafvorschriften . . . . . 795

§ 104. Krämermäkler . . . . . . . 796 
Nachtrag

Gesetz über die Kaufmannseigenschaft von

Handwerkern

Artikel 1 Nr. 1 und 2, Änderung des

\$1 Abs. 2 Nr. 2 u. 9 HGB. . . 796
Artikel 1 Nr. 3, Änderung des § 2 HGB. 797 Artikel 1 Nr. 4, Änderung des §4 H GB. 798 Artikel 2, Änderung des $\S 126$ FGG. 800 Artikel 3 . . . . . . . . . . . 800 Sachregister . . . . . . . 803 